Pacific Journal of Mathematics

CONTRACTION SEMI-GROUPS IN A FUNCTION SPACE 


\title{
CONTRACTION SEMI-GROUPS IN A FUNCTION SPACE
}

\author{
J. R. DORROH
}

Using the concepts of a semi inner-product and a dissipative operator, it is proven that if $X$ is a complex Banach space (under the supremum norm) of bounded complex valued functions on a set $S, p$ is a bounded positive function on $S$ which is bounded away from zero, $p X \subset X$, and $A$ is the infinitesimal generator of a strongly continuous (class $\left(C_{0}\right)$ ) semi-group of contraction operators in $X$, then $p A$ is also the infinitesimal generator of such a semi-group.

The notion of a semi inner-product was introduced by G. Lumer in [3].

DeFinition 1. A semi inner-product for a complex (real) Banach space $X$ is a function $[\cdot, \cdot]$ from $X \times X$ into the complex (real) numbers which satisfies

$$
\begin{gathered}
{[\alpha x+\beta y, z]=\alpha[x, z]+\beta[y, z],} \\
|[x, z]| \leqq\|x\| \cdot\|z\|,
\end{gathered}
$$

and

$$
[x, x]=\|x\|^{2} \text {. }
$$

There is at least one semi inner-product for every Banach space $X$, because we can define $[x, y]=f(x)$, where $f$ is a bounded linear functional on $X$ such that $\|f\|=\|y\|$, and $|f(y)|=\|y\|^{2}$ (see [4]).

By an operator in a Banach space $X$, we mean a linear transformation (not necessarily bounded) from a subspace of $X$ to a subspace of $X$. The notion of a dissipative operator in a Banach space is treated by G. Lumer and R. S. Phillips in [4].

Definition 2. An operator $A$ in a Banach space $X$ is said to be dissipative (with respect to a given semi inner-product for $X$ ) if

$$
\operatorname{re}[A x, x] \leqq 0
$$

for all $x$ in the domain of $A$.

By a contraction semi-group in a Banach space $X$ we mean a strongly continuous semi-group of contraction operators in $X$ which is of class $\left(C_{0}\right)$ (see [2]). A contraction operator in $X$ is a bounded linear transformation $T$ from $X$ into $X$ with $\|T\| \leqq 1$. Lumer and Phillips have given the following characterization [4, Theorem 3.1] of the infinitesimal generator of a contraction semi-group. 
THEOREM (Lumer and Phillips). Suppose $A$ is an operator in a Banach space $X$, the domain of $A$ is dense in $X$, and $[\cdot, \cdot \cdot]$ is a semi inner-product for $X$. Then $A$ is the infinitesimal generator of a contraction semi-group in $X$ if and only if $A$ is dissipative with respect to $[\cdot, \cdot]$, and the range of $I-A$ is all of $X$, where $I$ denotes the identity transformation on $X$.

THEOREM. Suppose $S$ is a set, $X$ is a complex Banach space (under the supremum norm) of bounded complex valued functions on $S, p$ is a bounded positive function on $S$ which is bounded away from zero, $p X \subset X$, and $A$ is the infinitesimal generator of $a$ contraction semi-group in $X$. Then $p A$ is also the infinitesimal generator of a contraction semi-group in $X$.

Proof. Let $U$ denote the Banach algebra of all bounded complex valued functions on $S$, and let $S_{1}$ denote the set of all nonzero multiplicative linear functionals on $S$. It follows from [1, pp. 272-277], especially [1, Corollary 19, p. 276], that

(i) if $m$ is in $S_{1}$, and $q$ is a nonnegative function in $U$, then $m(q) \geqq 0$, and

(ii) if $x$ is in $U$, then there is an $m$ in $S_{1}$ such that $|m(x)|=\|x\|$. For each $x$ in $X$, let $m_{x}$ denote an element $m$ of $S_{1}$ such that $|m(x)|=$ $\|x\|$, and for each $x, y$ in $X$, let

$$
[x, y]=m_{y}(x)\left[m_{y}(y)\right]^{*},
$$

where the $*$ denotes complex conjugation. Then $[\cdot, \cdot]$ is a semi innerproduct for $X$; it is the only one to be used from this point on. A dissipative operator in $X$ will mean one which is dissipative with respect to this semi inner-product.

If $q$ is a bounded nonnegative function on $S$, and $q X \subset X$, then

$$
\text { re }[q A x, x]=m_{x}(q) \text { re }[A x, x] \leqq 0,
$$

for all $x$ in $\mathfrak{D}(A)$, the domain of $A$, since $A$ is dissipative by [4, Theorem 3.1]. Therefore, $q A$ is dissipative. Also, the domain of $q A$ is $\mathfrak{D}(A)$, which is dense in $X$ by [2, Theorem 12.3.1, p. 360]. If

$$
\sup _{s \in S}|1-q(s)|<1 / 2,
$$

then $\|I-q\|$, the operator norm of $I-q$, is less than $1 / 2$, so that $I-q A$ is invertible, since

$$
I-q A=I-A+(I-q) A=\{I+(I-q) A R(1, A)\}(I-A),
$$

and 


$$
\|A R(1, A)\|=\|R(1, A)-I\| \leqq 2
$$

by $[2$, Theorem $12.3 .1, \mathrm{p} .360]$. Thus the range of $I-q A$ is all of $X$, and $q A$ generates a contraction semi-group in $X$ by [4, Theorem 3.1].

Since $F(p) X \subset X$ for every polynomial $F$, and $p$ is bounded and nonnegative, it follows from the classical Weierstrass theorem that $p^{(1 / n)} X \subset X$ for every positive integer $n$. Choose $n$ so that

$$
\sup _{s \in S}\left|1-[p(s)]^{(1 / n)}\right|<1 / 2,
$$

and let $r=p^{(1 / n)}$. This is possible because the range of $p$ is contained in a closed and bounded interval of positive numbers. By what was shown in the previous paragraph, $r A$ generates a contraction semigroup in $X$. If $1 \leqq j<n$, and $r^{j} A$ generates a contraction semi-group in $X$, then $r^{j+1} A$ does also, for

$$
r^{j+1} A=r\left(r^{j} A\right),
$$

and we can substitute $r$ for $q$ and $r^{j} A$ for $A$ in the argument given in the previous paragraph.

REMARK. An argument similar to the one given will establish the theorem if $X$ is taken to be a real Banach space (under the supremum norm) of bounded real valued functions on $S$, and the rest of the hypothesis remains the same. Also, we could take $A$ to be the generator of a class $\left(C_{0}\right)$ semi-group $[T(t) ; 0 \leqq t<\infty]$ of operators in $X$ such that for some $\omega>0$,

$$
\|T(t)\| \leqq e^{\omega t} \quad \text { for } t \geqq 0 .
$$

If

$$
\widetilde{T}(t)=e^{-\omega t} T(t) \quad \text { for } t \geqq 0,
$$

then $[\widetilde{T}(t)]$ is a contraction semi-group in $X$ and has the generator $\widetilde{A}=A-\omega$.

If

$$
V(t)=e^{\omega t p} \widetilde{V}(t) \quad \text { for } t \geqq 0,
$$

where $[\tilde{V}(t) ; 0 \leqq t<\infty]$ is the contraction semi-group generated by $p \widetilde{A}$, then $[V(t)]$ is a class $\left(C_{0}\right)$ semi-group of operators in $X$,

$$
\|V(t)\| \leqq e^{\omega t|| p \| \mid} \quad \text { for } t \geqq 0,
$$

and $[V(t)]$ is generated by $p A$. The author wishes to express his thanks to the referee for his suggestions. 


\section{REFERENCES}

1. N. Dunford and J. Schwartz, Linear Operators, Part I, Interscience, 1958.

2. E. Hille and R. S. Phillips, Functional analysis and semi-groups, Amer. Math. Soc. Colloquium Publication, 31, 1957.

3. G. Lumer, Semi inner-product spaces, Trans. Amer. Math. Soc. 100 (1961), 29-43. 4. G. Lumer and R. S. Phillips, Dissipative operators in a Banach space, Pacific J. Math. 11 (1961), 679-698.

Received October 19, 1965, and in revised form December 2, 1965. Presented to the Society, November 12,1965, under the title Contraction semi-groups in a function algebra.

Louisiana State University 


\section{PACIFIC JOURNAL OF MATHEMATICS}

\section{EDITORS}

\author{
H. SAMELSON \\ Stanford University \\ Stanford, California \\ J. P. JANS \\ University of Washington \\ Seattle, Washington 98105
}

\author{
J. DUGUNDJI \\ University of Southern California \\ Los Angeles, California 90007 \\ RICHARD ARENS \\ University of California \\ Los Angeles, California 90024
}

\section{ASSOCIATE EDITORS}
E. F. BECKENBACH
B. H. NeumanN
F. WOLF
K. YOSIDA

\section{SUPPORTING INSTITUTIONS}

\author{
UNIVERSITY OF BRITISH COLUMBIA \\ CALIFORNIA INSTITUTE OF TECHNOLOGY \\ UNIVERSITY OF CALIFORNIA \\ MONTANA STATE UNIVERSITY \\ UNIVERSITY OF NEVADA \\ NEW MEXICO STATE UNIVERSITY \\ OREGON STATE UNIVERSITY \\ UNIVERSITY OF OREGON \\ OSAKA UNIVERSITY \\ UNIVERSITY OF SOUTHERN CALIFORNIA
}

STANFORD UNIVERSITY

UNIVERSITY OF TOKYO

UNIVERSITY OF UTAH

WASHINGTON STATE UNIVERSITY

UNIVERSITY OF WASHINGTON

AMERICAN MATHEMATICAL SOCIETY CHEVRON RESEARCH CORPORATION TRW SYSTEMS

NAVAL ORDNANCE TEST STATION 


\section{Pacific Journal of Mathematics}

\section{Vol. 19, No. 1 \\ May, 1966}

A. R. Brodsky, The existence of wave operators for nonlinear equations... 1

Gulbank D. Chakerian, Sets of constant width................... 13

Robert Ray Colby, On indecomposable modules over rings with minimum condition....................................... 23

James Robert Dorroh, Contraction semi-groups in a function space ....... 35

Victor A. Dulock and Harold V. McIntosh, On the degeneracy of the Kepler

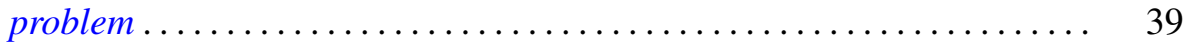

James Arthur Dyer, The inversion of a class of linear operators ......... 57

N. S. Gopalakrishnan and Ramaiyengar Sridharan, Homological dimension of Ore-extensions ................................. 67

Daniel E. Gorenstein, On a theorem of Philip Hall ................. 77

Stanley P. Gudder, Uniqueness and existence properties of bounded observables..................................... 81

Ronald Joseph Miech, An asymptotic property of the Euler function ....... 95

Peter Alexander Rejto, On the essential spectrum of the hydrogen energy and related operators ............................... 109

Duane Sather, Maximum and monotonicity properties of initial boundary

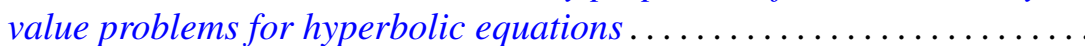

Peggy Strait, Sample function regularity for Gaussian processes with the parameter in a Hilbert space ........................... 159

Donald Reginald Traylor, Metrizability in normal Moore spaces ........... 175

Uppuluri V. Ramamohana Rao, On a stronger version of Wallis' formula ...............................

Adil Mohamed Yaqub, Some classes of ring-logics....... 